\title{
Renin-Angiotensin System Inhibitors Reduce Serum Asymmetric Dimethylarginine Levels and Oxidative Stress in Normotensive Patients with Chronic Kidney Disease
}

\author{
Hideki Fujii ${ }^{a}$ Keiji Kono ${ }^{a}$ Kentaro Nakai ${ }^{a}$ Shunsuke Goto ${ }^{a}$ \\ Riko Kitazawa $^{\text {b, c }}$ Masafumi Fukagawa ${ }^{a, d}$ Shinichi Nishi ${ }^{a}$
}

Divisions of a Nephrology and Kidney Center and ${ }^{b}$ Diagnostic Molecular Pathology, Kobe University Graduate School of Medicine, Kobe, 'Division of Molecular Pathology, Ehime University Graduate School of Medicine, Toon, and d Division of Nephrology and Metabolism, Department of Internal Medicine, Tokai University School of Medicine, Isehara, Japan

\section{Key Words}

Chronic kidney disease - Renin-angiotensin system inhibitor - Asymmetric dimethylarginine . Oxidative stress

\begin{abstract}
Background/Aims: The purpose of our study was to elucidate the relationship between asymmetric dimethylarginine (ADMA) and intrarenal lesions and to determine the effect of renin-angiotensin system inhibitors (RAS-Is) on serum ADMA levels, nitric oxide (NO) synthesis and oxidative stress in normotensive patients with chronic kidney disease (CKD). Methods: This study included 23 normotensive patients with chronic glomerulonephritis and normal or mildly impaired renal function who underwent renal biopsy. We evaluated the relationship between serum ADMA levels and intrarenal lesions, and examined renal function, urinary protein excretion, ADMA levels, NO synthesis, oxidative stress and blood pressure (BP) before and 3 months after starting the treatment with RAS-Is. Results: Serum ADMA levels were correlated only with arterial intimal fibroplastic thickness. Despite comparable renal function and $\mathrm{BP}$, serum $\mathrm{ADMA}$ levels and excretion of urinary protein excretion significantly decreased, and urinary NO metabolite excretion significantly increased after starting the treatment with RASIs. Oxidative stress markers also tended to be reduced by the treatment. Conclusion: These findings suggest that RAS-Is improve the NO system and decrease oxidative stress in normotensive patients with CKD. In addition, ADMA may be associated with intrarenal lesions and can be a useful marker for the effects of treatment in the early stages of CKD.
\end{abstract}


Fujii et al.: RAS-Is Reduce Serum Asymmetric Dimethylarginine Levels and Oxidative Stress in Normotensive Patients with Chronic Kidney Disease

\section{Introduction}

It is well known that chronic kidney disease (CKD) is a very important risk factor associated with cardiovascular events and mortality [1]. Therefore, its management is essential for improving patients' prognoses. Renin-angiotensin system inhibitors (RAS-Is) including angiotensin-converting enzyme inhibitor and angiotensin receptor blocker (ARB) can not only reduce urinary protein excretion and slow the decline of renal function but also prevent the progression of cardiovascular disease (CVD) in patients with CKD [2, 3]. Furthermore, basic and clinical reports have demonstrated that RAS-Is can suppress the activity of glomerular nephropathy. For example, an in vivo study showed that RAS-Is increase the permeability and size-selective functions of the glomerulus [4]. Furthermore, a clinical study of patients with IgA nephropathy demonstrated that RAS-Is significantly improve renal survival in proteinuric patients with normal or moderately reduced renal function [5]. Another study has reported that RAS-Is effectively reduce proteinuria and improve serum albumin in patients with lupus nephritis [6]. To summarize, RAS-Is are effective for the treatment of various types of CKD.

Asymmetric dimethylarginine (ADMA), an endogenous inhibitor of nitric oxide (NO) synthase, has been suggested to be a marker of endothelial dysfunction and atherosclerosis [7]. ADMA is also associated with the production of oxidative stress [8]. A previous study has reported that serum ADMA levels are increased in patients with CKD and are associated with renal function and proteinuria [9]. In addition, both experimental and clinical studies have demonstrated that RAS-Is decrease serum ADMA levels and improve endothelial dysfunction [10].

Although many studies have demonstrated the effect of RAS-Is in patients with CKD and hypertension, to the best of our knowledge, detailed data in normotensive patients with CKD are lacking. In the present study, we examined the effects of RAS-I treatment on oxidative stress and the NO system in normotensive patients with CKD.

\section{Subjects and Methods}

\section{Study Population}

Thirty-one normotensive patients with chronic glomerulonephritis who were hospitalized in our institution between January 2008 and December 2009 were enrolled in the present study. All patients met the criteria for normotensive subjects according to the guidelines of the Japanese Society of Hypertension. Patients who were taking RAS-Is or an immunosuppressant or who had a history of smoking, hypertension, diabetes mellitus, hyperlipidemia, overt infection, malignancy, inflammatory disease or CVD were excluded. Of the 31 patients, 8 were excluded from this study because of the discontinuation of prescribed medication, transfer to another facility or insufficient clinical data. For the 23 remaining patients, renal biopsies were performed after enrolment, and RAS-Is were administered for at least 3 months (losartan, $n=11$; olmesartan, $n=6$; valsartan, $n=5$, and enalapril, $n=1$ ). The patients did not take any other antihypertensive or renoprotective drugs, and there was no change in the medication dose during the study period. To compare the histological findings, 5 subjects with mild proteinuria whose renal histological findings showed only minor glomerular abnormalities were evaluated as controls. In addition, as for serum ADMA levels, 20 young healthy volunteers were evaluated. For the study patients, we prospectively performed blood and urinary examinations, renal histological analyses and blood pressure (BP) measurement before and 3 months after starting treatment with RAS-Is. The experimental protocols were approved by the appropriate institutional review committee and performed in accordance with the Helsinki Declaration of 1975, as revised in 2000. 
Table 1. Patients' baseline characteristics $(\mathrm{n}=23)$
Fujii et al.: RAS-Is Reduce Serum Asymmetric Dimethylarginine Levels and Oxidative Stress in Normotensive Patients with Chronic Kidney Disease

$\begin{array}{lc}\text { Age, years } & 48 \pm 4 \\ \text { Males/females } & 15 / 8 \\ \text { Systolic BP, mm Hg } & 115.6 \pm 3.1 \\ \text { Diastolic BP, mm Hg } & 67.6 \pm 2.3 \\ \text { Cr, mg/dl } & 0.99 \pm 0.07 \\ \text { eGFR, ml/min/1.73 m }{ }^{2} & 65.6 \pm 4.1 \\ \mathrm{BUN}, \mathrm{mg} / \mathrm{dl} & 15.2 \pm 1.0 \\ \mathrm{TP}, \mathrm{g} / \mathrm{dl} & 6.74 \pm 0.20 \\ \text { Albumin, g/dl } & 3.73 \pm 0.12 \\ \mathrm{ADMA}, \mathrm{nmol} / \mathrm{ml} & 0.46 \pm 0.01 \\ \mathrm{U}-8-\mathrm{OHdG}, \mathrm{ng} / \mathrm{mg} \bullet \mathrm{Cr} & 99.1 \pm 14.4 \\ \mathrm{U}-\mathrm{NOx} / \mathrm{U}-\mathrm{Cr} & 1.29 \pm 0.16 \\ \mathrm{U}-\mathrm{protein}, \mathrm{g} / \mathrm{g} \bullet \mathrm{Cr} & 1.22 \pm 0.32\end{array}$

$\mathrm{Cr}=$ Creatinine; eGFR = estimated glomerular filtration rate; $\mathrm{BUN}=$ blood urea nitrogen; $\mathrm{TP}=$ total protein; $\mathrm{U}-8-\mathrm{OHdG}=$ urinary $8-\mathrm{OHdG}$; $\mathrm{U}-\mathrm{Cr}=$ urinary creatinine; U-protein = urinary protein.

Table 2. Renal histological findings

\begin{tabular}{lccc}
\hline & Control $(\mathrm{n}=5)$ & CKD $(\mathrm{n}=17)$ & $\mathrm{p}$ value \\
\hline Global sclerosis, \% & 0 & $12.3 \pm 3.4$ & 0.038 \\
Interstitial fibrosis, \% & 0 & $13.4 \pm 3.7$ & 0.042 \\
Hyalinosis, \% & 0 & $5.3 \pm 4.0$ & 0.439 \\
Arterial intimal fibroplastic thickness, \% & $7.6 \pm 0.3$ & $22.0 \pm 3.4$ & 0.036 \\
8-OHdG-positive cell score & $42.1 \pm 6.7$ & $84.3 \pm 3.8$ & 0.032 \\
\hline
\end{tabular}

\section{Renal Histological Analysis}

Of the 23 study patients, renal biopsy specimens of 17 patients were available for histological analysis. We evaluated the extent of glomerulosclerosis, interstitial fibrosis, arteriolar hyalinosis and intimal fibroplastic thickness of interlobular arteries in a blinded manner. The number of globally sclerotic glomeruli was counted to determine the extent of global sclerosis. Furthermore, formation of 8-hydroxydeoxyguanosine (8-OHdG) was assessed with anti-8-OHdG monoclonal antibodies raised in humans (NOF Corp., Tokyo, Japan). 8-OHdGpositive cells in all glomeruli were counted, and the average was used as the 8-OHdG-positive cell score. The following formulae were used to calculate global sclerosis, interstitial fibrosis, arteriolar hyalinosis and arterial intimal fibroplastic thickness, respectively:

global sclerosis $(\%)=100 \times$ (number of globally sclerotic glomeruli/total number of glomeruli),

interstitial fibrosis $(\%)=100 \times$ (area of fibrosis/total area of the specimens),

arteriolar hyalinosis $(\%)=100 \times$ (number of arterioles with hyalinization/total number of arterioles), and

arterial intimal fibroplastic thickness $(\%)=100 \times(a+b) / o d$, where $a$ and $b=$ intimal thickness of interlobular arteries and od $=$ outer diameter.

\section{Serum and Urine Measurements}

Before and 3 months after starting treatment with RAS-Is, venous blood was collected from study patients in the morning following overnight fasting. Urinary NO metabolites (U-NOx) and 8-OHdG excretion were determined using ELISA kits (NOx: Dojindo Laboratories, Kumamoto, Japan; 8-OHdG: Japan Institute for Control of Aging, Shizuoka, Japan). Serum ADMA 
Fujii et al.: RAS-Is Reduce Serum Asymmetric Dimethylarginine Levels and Oxidative Stress in Normotensive Patients with Chronic Kidney Disease

Fig. 1. Comparison of serum ADMA levels between healthy subjects and patients with CKD.
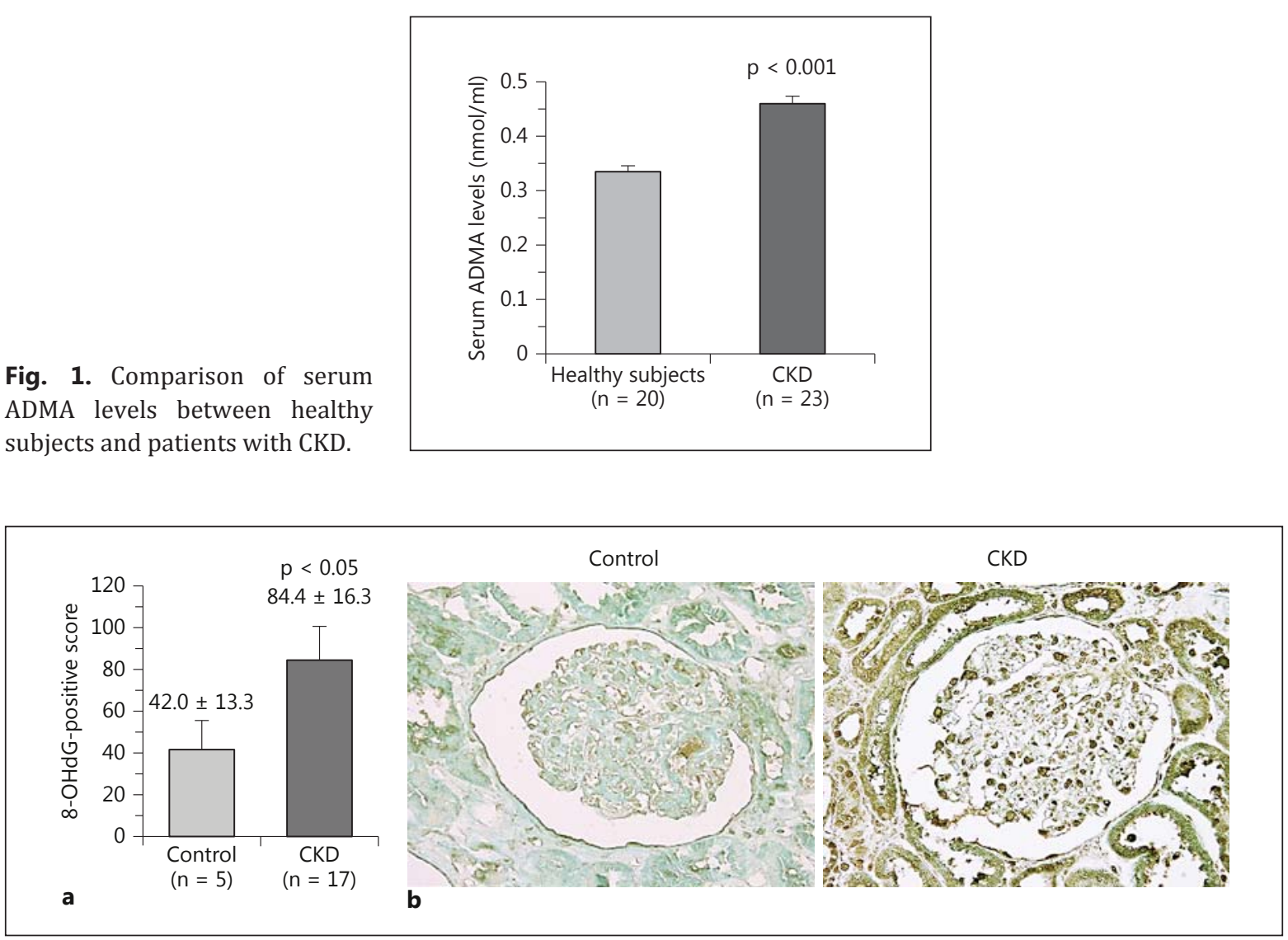

Fig. 2. Histological examination of the kidney sections for 8-OHdG. a 8-OHdG-positive cell scores were significantly higher in the patients with CKD than in the control subjects. b Immunostaining for 8-OHdG is indicated by brown reaction products.

levels were determined using a novel high-performance liquid chromatography method. Other laboratory tests were conducted using standardized clinical laboratory methods.

\section{Statistical Analysis}

We used the computer software application StatView 5.0 (SAS Institute, Cary, N.C., USA) for all statistical analyses. Values are presented as mean \pm SEM. For continuous variables, the Mann-Whitney $U$ test was used to analyze the significance of the differences between the 2 groups. Pearson's correlation coefficient was used to analyze the relationship between the variables. $\mathrm{p}<0.05$ was considered to be statistically significant.

\section{Results}

\section{Patients' Characteristics and Renal Histology}

The baseline characteristics of the study patients are shown in table 1. Serum ADMA levels in the study patients were higher than those in the healthy subjects (fig. 1). Renal histological analysis revealed more severe lesions in the study patients compared with the control subjects (table 2). Furthermore, the 8-OHdG-positive cell score was significantly higher in the patients with CKD than in the control subjects (fig. 2). 
Table 3. Relationship between serum ADMA levels and clinical characteristics
Table 4. Relationship between serum ADMA levels and renal histological findings
Fujii et al.: RAS-Is Reduce Serum Asymmetric Dimethylarginine Levels and Oxidative Stress in Normotensive Patients with Chronic Kidney Disease

\begin{tabular}{lrl}
\hline & $r$ & $p$ value \\
\hline Age & 0.362 & 0.117 \\
Sex & 0.298 & 0.203 \\
Systolic BP & 0.013 & 0.933 \\
Diastolic BP & 0.165 & 0.272 \\
Cr & 0.274 & 0.065 \\
eGFR & -0.336 & 0.024 \\
BUN & 0.375 & 0.010 \\
TP & 0.050 & 0.833 \\
Albumin & 0.015 & 0.950 \\
U-8-OHdG & 0.219 & 0.163 \\
U-NOx/U-Cr & -0.160 & 0.313 \\
U-protein & 0.275 & 0.064 \\
\hline
\end{tabular}

$\mathrm{Cr}=$ Creatinine; $\mathrm{eGFR}=$ estimated glomerular filtration rate; $\mathrm{BUN}=$ blood urea nitrogen; $\mathrm{TP}=$ total protein; $\mathrm{U}-8-\mathrm{OHdG}=$ urinary $8-\mathrm{OHdG}$; $\mathrm{U}-\mathrm{Cr}=$ urinary creatinine; U-protein = urinary protein.

\begin{tabular}{lll}
\hline & $\mathrm{r}$ & $\mathrm{p}$ value \\
\hline Global sclerosis & 0.205 & 0.415 \\
Interstitial fibrosis & 0.309 & 0.212 \\
Hyalinosis & 0.092 & 0.910 \\
Arterial intimal fibroplastic thickness & 0.573 & 0.013 \\
\hline
\end{tabular}

Correlation of ADMA with Clinical Characteristics and Renal Histology

We evaluated the correlation of serum ADMA levels with the patients' clinical characteristics (table 3) and renal histological findings (table 4). Serum ADMA levels were significantly correlated with estimated glomerular filtration rate and tended to be correlated with the degree of urinary protein excretion. Among the renal histological findings, we found that only arterial intimal fibroplastic thickness was significantly related to serum ADMA levels.

Changes of ADMA, NO and 8-OHdG by RAS-Is

Although BP and renal function did not differ significantly during the study period, the degree of urinary protein excretion was significantly reduced 3 months after starting treatment with RAS-Is (fig. 3). In addition, serum ADMA levels and U-NOx and urinary 8-OHdG excretion were improved 3 months after starting treatment with RAS-Is compared with the respective values at baseline (fig. 4).

\section{Discussion}

Our study demonstrated that serum ADMA levels were significantly higher in the normotensive patients with CKD than in the control subjects, that arterial intimal fibroplastic thickness was significantly correlated with serum ADMA levels and that RAS-Is lowered the elevated serum ADMA levels and oxidative stress and increased NO production.

The mechanisms underlying abnormalities in CKD are complicated, and several factors contribute to their pathogenesis. Of these factors, oxidative stress is considered to play a key 
Fujii et al.: RAS-Is Reduce Serum Asymmetric Dimethylarginine Levels and Oxidative Stress in Normotensive Patients with Chronic Kidney Disease

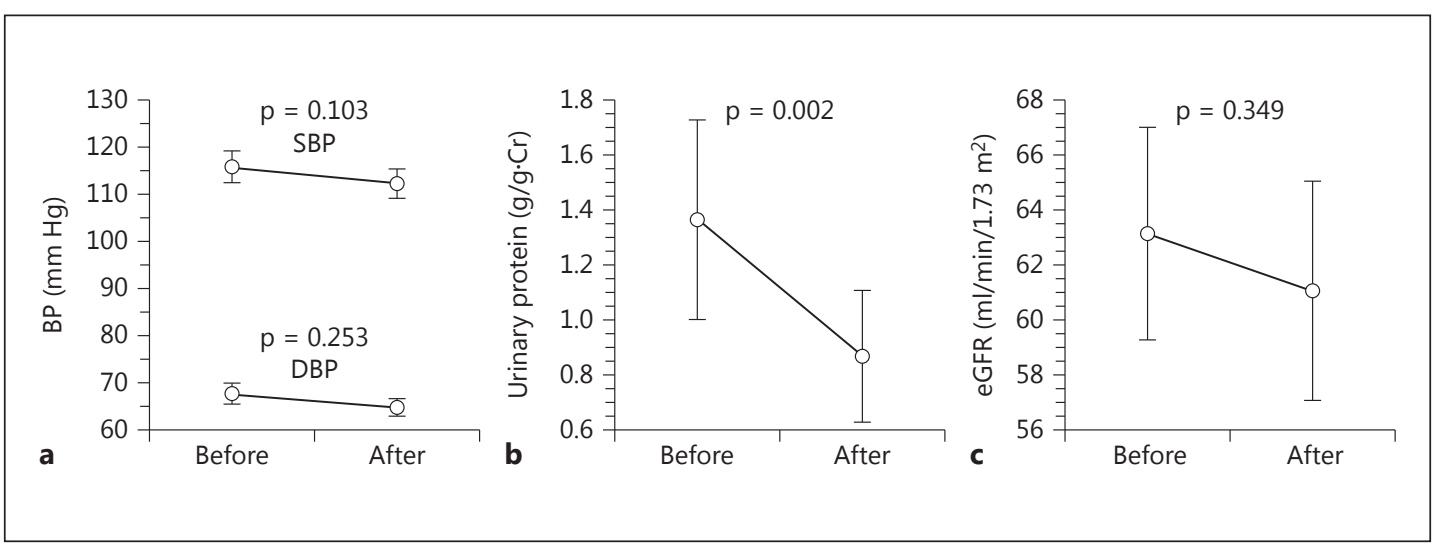

Fig. 3. Changes in systolic (SBP) and diastolic BP (DBP) (a), urinary protein excretion (b) and renal function after starting treatment with RAS-Is (c). eGFR = Estimated glomerular filtration rate; before = before treatment; after = after treatment.

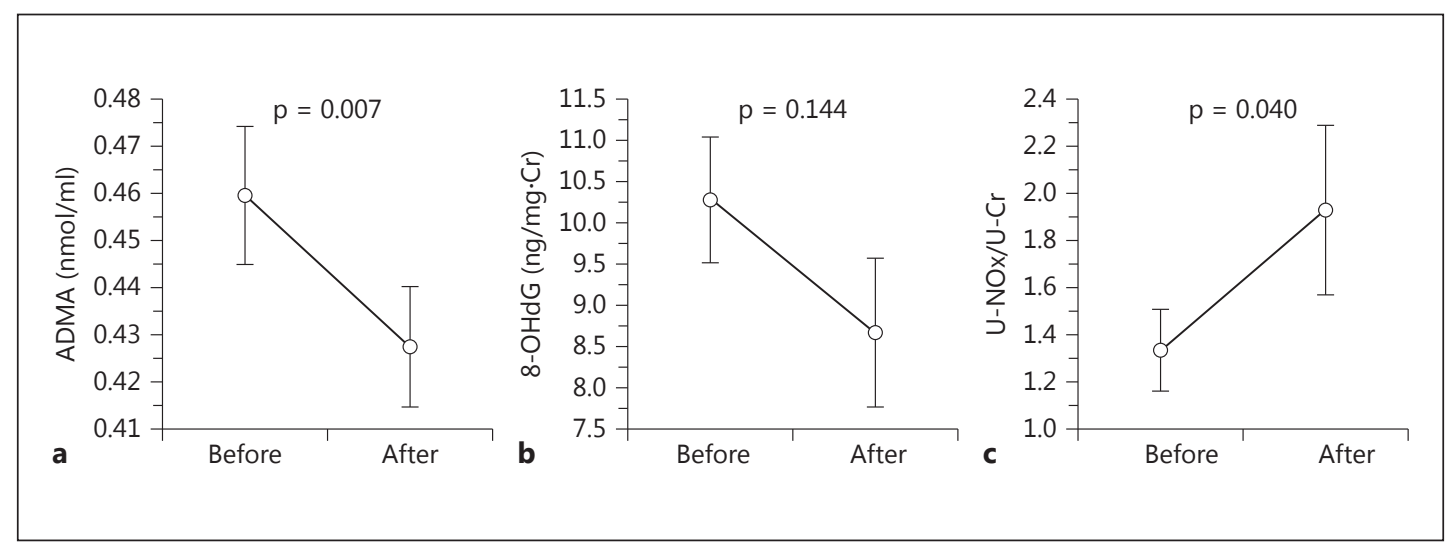

Fig. 4. Changes in ADMA (a), U-NOx (b) and oxidative stress (c) after starting treatment with RAS-Is. U-Cr = Urinary concentration of creatinine.

role in the progression of CKD $[11,12]$. In the present study, compared with healthy subjects, systemic and intrarenal oxidative stress increased in normotensive patients with CKD. These findings suggested a relationship between oxidative stress and the progression of CKD in such patients.

ADMA is an endogenous competitive inhibitor of NO synthase, and NO is a potent antiatherosclerotic molecule [7]. Therefore, increased ADMA levels lead to a decrease in NO synthesis and progressive damage due to impaired vascular function in the kidneys, heart and other systemic organs. Elevated ADMA levels are associated not only with endothelial dysfunction $[8,13,14]$ but also with increased oxidative stress $[15,16]$, thereby linking vascular function and redox mechanisms in CKD and CVD. In addition, serum ADMA levels have been reported to be markers of endothelial dysfunction and/or atherosclerosis [17] and to be associated not only with renal function but also with proteinuria in CKD $[8,18]$. The results of our study demonstrated that serum ADMA levels were significantly correlated with the intrarenal vascular lesions even in normotensive patients with CKD. With regard to the clinical data, serum ADMA levels were correlated with renal function and proteinuria. Taking 
these results into account, the increase of serum ADMA levels is believed to be the first step in the process of the progression of CKD. Therefore, these findings suggested that serum ADMA levels reflect the severity of CKD and could be used as a surrogate marker for the progression of CKD.

Many reports have shown the efficacy of RAS-Is in renal diseases. Angiotensinconverting enzyme inhibitor and ARB have been shown to reduce urinary protein excretion and slow the progression of renal dysfunction $[2,3]$. However, only few studies have demonstrated the efficacy of RAS-Is in normotensive patients with CKD in clinical settings. Makino et al. [19] reported that ARB reduces the transition to overt nephropathy in normotensive diabetic patients, suggesting that ARB has favorable BP-independent effects on CKD. Web et al. $[20,21]$ demonstrated that RAS-Is effectively reduced proteinuria in normotensive children with CKD.

Angiotensin II, which acts on angiotensin II type 1 receptors, increases oxidative stress, in part by activating nicotinamide adenine dinucleotide phosphate oxidase and ADMA, which in turn activates the local renin-angiotensin system [15, 22, 23]. Therefore, RAS-Is can lower oxidative stress, which has been suggested to be associated with a reduction in serum ADMA levels independently of lowering the BP. In patients with CKD, RAS-Is has been reported to reduce ADMA, proteinuria levels and oxidative stress [24, 25]. However, no study has actually demonstrated that RAS-Is can reduce ADMA levels in normotensive patients with CKD.

The main limitation of our study was the small number of study patients. However, our patients were carefully and appropriately treated and we closely observed them prospectively and longitudinally. Although only few prospective and longitudinal studies have elucidated the detailed mechanisms of the progression of CKD on this topic in the early stages of CKD, we performed both a biochemical analysis and histological evaluations. Therefore, we consider the results of the present study to be valuable for understanding the mechanisms and effects of RAS-Is on the progression of CKD. Another limitation was that the present study included a group of patients with heterogeneous renal etiologies. However, all study patients were normotensive and relatively young. Other patient characteristics were very similar. In the near future, we plan to conduct a randomized controlled trial with a large number of study patients to clarify these details.

Our data suggest that RAS-Is prevent the progression of chronic glomerulonephritis by reducing serum ADMA levels and oxidative stress in normotensive patients with CKD. In addition, the findings suggest that ADMA may be associated with intrarenal lesions and can be used as a useful surrogate marker for the effects of treatment in the early stages of CKD.

\section{Acknowledgement}

This study was in part presented at the annual meeting of the European Renal Association - European Dialysis and Transplant Association Congress, 2011.

\section{Disclosure Statement}

None of the authors have any conflict of interest to report. 
Fujii et al.: RAS-Is Reduce Serum Asymmetric Dimethylarginine Levels and Oxidative Stress in Normotensive Patients with Chronic Kidney Disease

\section{References}

1 Go AS, Chertow GM, Fan D, McCulloch CE, Hsu CY: Chronic kidney disease and the risks of death, cardiovascular events, and hospitalization. N Engl J Med 2004;351:1296-1305.

2 Ruggenenti P, Perna A, Gherardi G, Garini G, Zoccali C, Salvadori M, Scolari F, Schena FP, Remuzzi G: Renoprotective properties of ACE-inhibition in non-diabetic nephropathies with non-nephrotic proteinuria. Lancet 1999;354:359-364.

3 Brenner BM, Cooper ME, de Zeeuw D, Keane WF, Mitch WE, Parving HH, Remuzzi G, Snapinn SM, Zhang Z, Shahinfar S; RENAAL Study Investigators: Effects of losartan on renal and cardiovascular outcomes in patients with type 2 diabetes and nephropathy. N Engl J Med 2001;345:861-869.

4 Remuzzi A, Puntorieri S, Battaglia C, Bertani T, Remuzzi G: Angiotensin converting enzyme inhibition ameliorates glomerular filtration of macromolecules and water and lessens glomerular injury in the rat. J Clin Invest 1990;85:541-549.

5 Praga M, Gutiérrez E, González E, Morales E, Hernández E: Treatment of IgA nephropathy with ACE inhibitors: a randomized and controlled trial. J Am Soc Nephrol 2003;14:1578-1583.

6 Tse KC, Li FK, Tang S, Tang CS, Lai KN, Chan TM: Angiotensin inhibition or blockade for the treatment of patients with quiescent lupus nephritis and persistent proteinuria. Lupus 2005;14:947-952.

7 Cooke JP, Tsao PS: Is NO an endogenous antiatherogenic molecule? Arterioscler Thromb 1994;14:653-655.

-8 Fujii H, Takiuchi S, Kawano Y, Fukagawa M: Putative role of asymmetric dimethylarginine in microvascular disease of kidney and heart in hypertensive patients. Am J Hypertens 2008;21:650-656.

-9 Fu YF, Xiong Y, Guo Z: A reduction of endogenous asymmetric dimethylarginine contributes to the effect of captopril on endothelial dysfunction induced by homocysteine in rats. Eur J Pharmacol 2005;508:167-175.

10 Delles C, Schneider MP, John S, Gekle M, Schmieder RE: Angiotensin converting enzyme inhibition and angiotensin II AT1-receptor blockade reduce the levels of asymmetrical N(G), N(G)-dimethylarginine in human essential hypertension. Am J Hypertens 2002;15:590-593.

11 Cottone S, Lorito MC, Riccobene R, Nardi E, Mulè G, Buscemi S, Geraci C, Guarneri M, Arsena R, Cerasola G: Oxidative stress, inflammation and cardiovascular disease in chronic renal failure. J Nephrol 2008;21:175-179.

12 Shah SV, Baliga R, Rajapurkar M, Fonseca VA: Oxidants in chronic kidney disease. J Am Soc Nephrol 2007;18: 16-28.

13 Juonala M, Viikari JS, Alfthan G, Marniemi J, Kahonen M, Taittonen L, Laitinen T, Raitakari OT: Brachial artery flow-mediated dilation and asymmetrical dimethylarginine in the cardiovascular risk in young Finns study. Circulation 2007;116:1367-1373.

14 Antoniades C, Demosthenous M, Tousoulis D, Antonopoulos AS, Vlachopoulos C, Toutouza M, Marinou K, Bakogiannis C, Mavragani K, Lazaros G, Koumallos N, Triantafyllou C, Lymperiadis D, Koutsilieris M, Stefanadis C: Role of asymmetrical dimethylarginine in inflammation-induced endothelial dysfunction in human atherosclerosis. Hypertension 2011;58:93-98.

15 Luo Z, Teerlink T, Griendling K, Aslam S, Welch WJ, Wilcox CS: Angiotensin II and NADPH oxidase increase ADMA in vascular smooth muscle cells. Hypertension 2010;56:498-504.

-16 Antoniades C, Shirodaria C, Leeson P, Antonopoulos A, Warrick N, Van-Assche T, Cunnington C, Tousoulis D, Pillai R, Ratnatunga C, Stefanadis C, Channon KM: Association of plasma asymmetrical dimethylarginine (ADMA) with elevated vascular superoxide production and endothelial nitric oxide synthase uncoupling: implications for endothelial function in human atherosclerosis. Eur Heart J 2009;30:1142-1150.

17 Miyazaki H, Matsuoka H, Cooke JP, Usui M, Ueda S, Okuda S, Imaizumi T: Endogenous nitric oxide synthase inhibitor. A novel marker of atherosclerosis. Circulation 1999;99:1141-1146.

18 Fliser D, Kronenberg F, Kielstein JT, Morath C, Bode-Böger SM, Haller H, Ritz E: Asymmetric dimethylarginine and progression of chronic kidney disease: the mild to moderate kidney disease study. J Am Soc Nephrol 2005; 16:2456-2461.

19 Makino H, Haneda M, Babazono T, Moriya T, Ito S, Iwamoto Y, Kawamori R, Takeuchi M, Katayama S; INNOVATION Study Group: Prevention of transition from incipient to overt nephropathy with telmisartan in patients with type 2 diabetes. Diabetes Care 2007;30:1577-1578.

20 Webb NJ, Lam C, Loeys T, et al: Randomized, double-blind, controlled study of losartan in children with proteinuria. Clin J Am Soc Nephrol 2010;5:417-424.

-21 Webb NJ, Shahinfar S, Wells TG, Massaad R, Gleim GW, Santoro EP, Sisk CM, Lam C: Losartan and enalapril are comparable in reducing proteinuria in children. Kidney Int 2012;82:819-826.

22 Veresh Z, Racz A, Lotz G, Koller A: ADMA impairs nitric oxide-mediated arteriolar function due to increased superoxide production by angiotensin II-NAD(P)H oxidase pathway. Hypertension 2008;52:960-966.

23 Chabrashvili T, Kitiyakara C, Blau J, Karber A, Aslam S, Welch WJ, Wilcox CS: Effects of ANG II type 1 and 2 receptors on oxidative stress, renal NADPH oxidase, and SOD expression. Am J Physiol Regul Integr Comp Physiol 2003;285:R117-R124.

24 Yilmaz MI, Sonmez A, Saglam M, Yaman H, Cayci T, Kilic S, Eyileten T, Caglar K, Oguz Y, Vural A, Yenicesu M, Axelsson J: Reduced proteinuria using ramipril in diabetic CKD stage 1 decreases circulating cell death receptor activators concurrently with ADMA. A novel pathophysiological pathway? Nephrol Dial Transplant 2010;25:3250-3256.

25 Aslam S, Santha T, Leone A, Wilcox C: Effects of amlodipine and valsartan on oxidative stress and plasma methylarginines in end-stage renal disease patients on hemodialysis. Kidney Int 2006;70:2109-2115. 